\title{
A Systems Study of An RF Power Source For A 1 TeV Next Linear Collider Based Upon the Relativistic-Klystron Two-Beam Accelerator*
}

\author{
S. Yu1 , F. Deadrick ${ }^{2}$, N. Goffeney1, E. Henestroza ${ }^{1}$, T. Houck ${ }^{2}$, \\ H. Li ${ }^{1}$, C. Peters ${ }^{1}$, L. Reginato ${ }^{1}$, A. Sessler ${ }^{1}$, \\ D. Vanecek ${ }^{1}$ and G. Westenskow ${ }^{2}$ \\ ${ }^{1}$ Lawrence Berkeley Laboratory \\ University of California, Berkeley, California 94720 \\ ${ }^{2}$ Lawrence Livermore National Laboratory \\ University of California, Livermore, California 94550
}

\begin{abstract}
A systems study, including physics, engineering and costing, has been conducted to assess the feasibility of a relativistic-klystron two-beam-accelerator (RKTBA) system as a RF power source candidate for a $1 \mathrm{TeV}$ linear collider. Several key issues associated with a realizable RK-TBA system have been addressed, and corresponding schemes have been developed and examined quantitatively. A point design example has been constructed to present a concrete conceptual design which has acceptable transverse and longitudinal beam stability properties. The overall efficiency of RF production for such a power source is estimated to be $36 \%$, and the cost of the full system is estimated to be less than 1 billion dollars.
\end{abstract}

\section{OVERVIEW}

\subsection{TBA Issues}

As a power source for linear colliders. The main advantage of two-beamaccelerator (1) is high efficiency for drive-beam to if power conversion. Experimentally, very high peak powers have been achieved in free-electron-laser (2) and relativistic-klystron (3) devices. The basic concept of reacceleration was demonstrated in recent experiments at LLNL (4).

These experimental successors not withstanding, several critical issues must be addressed if two-beam accelerators are to be viable as practical power sources for future linear colliders.

The general concerns about relativistic-klystron two-beam accelerators (5) (RK-TBA) as a realizable power source evolve around 3 issues:

* Work supported in part by the Director, Office of Energy Research, Office of High Energy and Nuclear Physics, Division of High Energy Physics, of the US Department of Energy under contract(s) No. DE-AC03-76SF00098 at Lawrence Berkeley Laboratory and W-7405-ENG-48 at Lawrence Livermore National Laboratory.
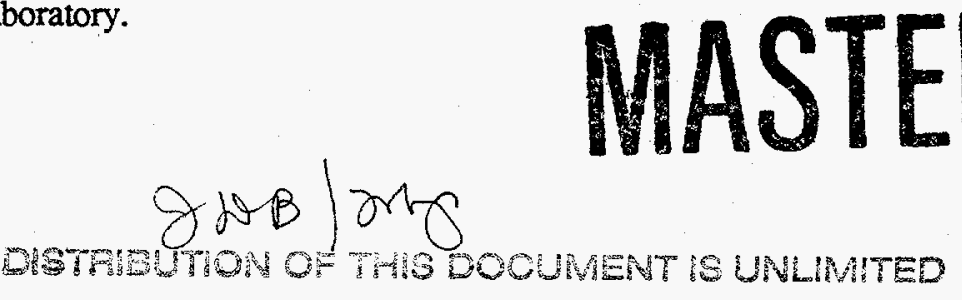
1. The induction linac technology is not as mature as existing rf-klystron technology.

2. The TBA beam dynamics, particularly BBU, is difficult.

3. Induction linacs are perceived to be very expensive.

The objective and the approach undertaken in the present study were motivated by these concerns.

In regards to the cost issue, past cost estimates were based on the technology of ETAII (6) and ATA (7), which are applications requiring short pulse (50-70 ns) high repetition rate, $(\mathrm{kHz})$ high current $(\sim 10 \mathrm{kA})$. We have attempted in this study to design an induction linac specifically for the RK-TBA.

In regards to the beam dynamics issues, we offer in this design conceptual solutions for longitudinal beam stability as well as BBU control for both the low frequency component, associated with induction gaps, and the high frequency component, associated with rf extraction cavities. These concepts are supported by detailed simulations.

Finally, in regards to the technology maturity issue, we note that significant advances in induction linac technology have already been made and are being made in intense electron beam $(6,7)$ and heavy ion fusion (8) programs. What is required is technology development specifically tailored to linear collider power source issues.

With these general considerations in mind, we propose a new version of the RK-TBA. A systems study (9), including physics and engineering designs, as well as "bottom-up" costing, has been conducted for a point design example with the RK-TBA as a power source for a $1 \mathrm{TeV}$ center-of-mass Next Linear Collider (NLC) (10).

\subsection{The Key Ideas}

To address the critical TBA issues quantitatively is the subject matter of this report. A few key ideas underlie the whole design and are summarized below:

1. 'Betatron node scheme' for high frequency BBU control -- The most severe $\mathrm{BBU}$ instability is associated with the dipole mode (HEM 11 mode) in the rf cavities. Strong suppression of this mode is achieved by introducing a 'betatron node scheme' in which adjacent rf extraction cavities are placed exactly one betatron period apart. This scheme minimizes beam centroid displacement which excites the dipole mode, and alters the nature of the instability from exponential to a slow secular growth.

2. Landau damping for low frequency BBU suppression -- Quite apart from the high frequency dipole mode associated with the rf cavities, there is a low frequency (a few $\mathrm{GHz}$ ) dipole mode associated with the induction reacceleration gaps. This BBU instability is ameliorated by Landau damping due to a large energy spread inherent in the rf buckets of the bunched drive beam. Combining this feature with dipole mode suppression measures in the induction gap design, the calculated low frequency BBU growth is minimal.

3. Inductively detuned cavities for longitudinal beam stability -- To maintain tight rf bunches over long distances with multiple extraction cavities, the rf output structures are inductively detuned. While the concept of inductive detuning is not new, the theoretical framework has to be developed and implemented in 


\section{DISCLAIMER}

This report was prepared as an account of work sponsored by an agency of the United States Government. Neither the United States Government nor any' agency thereof, nor any of their employees, make any warranty, express or implied, or assumes any legal liability or responsibility for the accuracy, completeness, or usefulness of any information, apparatus, product, or process disclosed, or represents that its use would not infringe privately owned rights. Reference herein to any specific commercial product, process, or service by trade name, trademark, manufacturer, or otherwise does not necessarily constitute or imply its endorsement, recommendation, or favoring by the United States Government or any agency thereof. The views and opinions of authors expressed herein do not necessarily state or reflect those of the United States Government or any agency thereof. 


\section{DISCLAIMER}

Portions of this document may be illegible in electronic image products. Images are produced from the best available original document. 
simulation codes for traveling wave output structures. Cavity parameters required for stable beam propagation through multi structures were determined.

4. Small low-cost induction cells -- The induction cells in the present design are about 1 foot in diameter, much smaller than most of the known induction machines. This design was possible because of three key features:

(a) Use of Metglas ${ }^{\mathrm{TM}}$ for the induction cores. This is a low-cost magnetic material that can accommodate a large flux swing;

(b) Use of low-field permanent magnet quadrupoles. The relatively high drive beam energy of $10 \mathrm{MeV}$ (compared to klystron beams) is a natural match to a strong focusing transport system with low-cost ferrites. An additional advantage of permanent magnets is, of course, the elimination of power supplies;

(c) Narrow beam pipes -- the design pipe diameter of $5 \mathrm{~cm}$ is much smaller than other existing high current machines. This is possible because of the relatively low current (by induction machine standards) of $600 \mathrm{amps}$, and the low frequency BBU suppression features described earlier (large beam pipes in usual induction machines are dictated by BBU considerations).

5. Low-voltage pulse power system for efficiency and cost -- The induction cell design matches naturally a $20 \mathrm{kV}$ system with pulse forming networks triggered by ceramic thyratrons and powered by DC power supplies and command resonant charging systems. This system requires no step-up transformers, and eliminates losses associated with going to a high voltage system.

\subsection{Systems Study Approach}

The objective of the present study is to assess the technical feasibility as well as the cost and efficiency of a TBA-based system. To achieve this goal, we felt that it was essential to be reasonably concrete. For this reason, we have chosen for our study a TBA power source for the NLC Upgrade considered by SLAC. The present thinking about the Next Linear Collider is a $15 \mathrm{~km}$ machine with a center-of-mass energy of $500 \mathrm{GeV}$ powered by X-band klystrons and if pulse compression systems. This machine should be upgradeable to $1 \mathrm{TeV}$ center-ofmass. Our study considers the TBA as a power source candidate for the $1 \mathrm{TeV}$ version of the NLC.

The engineering and costing efforts in the present study draw heavily from recent work in Heavy Ion Fusion (HIF). The U.S. effort in HIF is focused on the induction accelerator approach. Research activities in the past few years have centered around the Induction Linac Scaled Experiments (ILSE). Substantial engineering activities were committed to the ILSE CDR, and a $2 \mathrm{MV}$ heavy ion injector at full driver scale was constructed and successfully operated in 1993. In addition, an engineering and costing study was performed recently for a recirculator version of the induction fusion driver. The present TBA study leverages against past HIF experience with many design features as well as engineering and costing expertise from the ILSE, heavy ion injector, and recirculator studies.

A first engineering and costing exercise for the full TBA system has been performed. The electrical design includes all components starting from the AC power distribution system, to the DC power supplies, the command resonant charging system, the pulse forming networks, and the induction cores. Racks and installation, as well as instrumentation and control, were included in this exercise. The mechanical design and costing includes details of the induction cells, if 
structures, vacuum, alignment, and utilities. Costs were estimated with a 'bottomup' approach, assuming mass production procedures for fabrication and assembly.

The physics study utilizes a number of simulation codes developed in the past few years in connection with the TBA experimental program at LLNL. The 1-D and 2-D longitudinal beam dynamics codes RKS have been used for the design of TBA experiments as well as the modeling of a number of other klystron experiments at SLAC and VLEPP, and code results were generally consistent with the measurements. The BBU code OMICE includes regenerative as well as cumulative effects, and code results agree with BBU observed in the TBA experiments. RKS and OMICE were used extensively for the physics design. For cavity design, we use URMEL and MAFIA, as well as the induction cell design code AMOS developed at LLNL.

\subsection{The Key Results}

We have constructed a point design of a power source for a $1 \mathrm{TeV}$ NLC that consists of 50 independent RK-TBA units, each $300 \mathrm{~m}$ long. End-to-end modeling with the RKS code demonstrates stable rf output over the entire RKTBA unit. BBU simulations with OMICE show acceptable high frequency as well as low frequency BBU growth over the whole $300 \mathrm{~m}$. Sensitivity of these results to beam and cavity parameters were studied. The cavity parameters for the beam dynamics simulations were calculated with URMEL, MAFIA, and AMOS.

Conceptual engineering designs were performed for all electrical and mechanical components from wall plug to rf input to the high gradient accelerating structures. Our preliminary cost estimate for the TBA-based power source for a $1 \mathrm{TeV}$ c.m. NLC is about $\$ 1$ billion. This estimate includes the fabrication and assembly of all components, as well as Engineering, Design, and Integration. Conventional facilities and institutional overhead are considered as part of the main NLC project, and are not separately costed in this power source engineering and costing exercise. The overall efficiency of the system (wall plug to rf ) is estimated to be $36 \%$.

\section{POINT DESIGN EXAMPLE}

\subsection{Power Source Requirements}

The objective of the present study is to construct a conceptual design of the power source for the $1 \mathrm{TeV}$ NLC. Our philosophy is to stay within the design constraints of the NLC and to have a power source system that matches the high gradient structures being considered for the NLC. However, since the parameters of the $1 \mathrm{TeV}$ NLC have not been finalized, we need to make some choices for "power source requirements" in order to make design and costing studies concrete. The design goals for the present study are as follows: 


\author{
If frequency \\ repetition rate \\ peak power/cavity \\ distance between extraction cavities \\ pulse length (flat-top) \\ pulse rise time
}

\author{
$11.424 \mathrm{GHz}$ \\ $120 \mathrm{~Hz}$ \\ $360 \mathrm{MW}$ \\ $2 \mathrm{~m}$ \\ $200 \mathrm{~ns}$ \\ $125 \mathrm{~ns}$
}

The peak power of $360 \mathrm{MW}$ at $4 \mathrm{X}$ SLAC frequency corresponds to an unloaded gradient of $100 \mathrm{MV} / \mathrm{m}$ in high gradient structures presently tested at SLAC. This power is generated from an extraction cavity in the TBA every $2 \mathrm{~m}$, to match the $1.8 \mathrm{~m}$ accelerator sections, and to provide adequate spacing in between the HGS's for input and output couplers, focusing etc. In this study, we will be costing the power source for a total of $15 \mathrm{~km}$ of this high gradient structure, $7.5 \mathrm{~km}$ for each arm of the linear collider. There is a total of 7500 of these extraction cavities, with a total peak power of 2.7 TW. The unloaded energy gain from each arm of the linear collider is $675 \mathrm{GeV}$. Assuming a loaded gradient that is $75 \%$ of the unloaded, the final energies of the electron and positron bunches are $506 \mathrm{GeV}$ each.

The pulse length is determined by the fill time of the structure plus the length of the multi-bunch particle train. In order to maintain constant particle energy from bunch to bunch, the required electric-field should grow linearly in the first $100 \mathrm{~ns}$, and remain constant over the remainder of the beam-on time. The flat-top is specified as $200 \mathrm{~ns}$, which allows us to accelerate pulse trains with twice the number of bunches in the $1 \mathrm{TeV}$ NLC than the present SLAC parameter list ( $100 \mathrm{~ns}$ flat-top), leading to a four-fold increase in luminosity. This is possible for our TBA design at only modest cost increase, and is a natural extension of the NLC parameter set to take advantage of the RK-TBA structure. Because of the intrinsically high efficiency of the RK-TBA, the average power required stays at about $200 \mathrm{MW}$.

The required electric field at the front of the $100 \mathrm{~ns}$ rise is roughly $25 \%$ of the asymptotic value. Hence, we specify our input pulse to rise linearly (in field) from zero to its full value in $125 \mathrm{~ns}$. The power increases quadratically over the rise time. This pulse shape can be generated in an RK-TBA by a corresponding linear rise in the current waveform of the drive beam, and a linear rise in the reacceleration voltage as well. Further refinement of the pulse shape to match dispersion in the high-gradient structure and to further improve energy flatness can be achieved by appropriate shaping of the drive beam current and voltage, but are not considered in the present study.

\subsection{RK-TBA Architecture}

To power a $15 \mathrm{~km}$ long NLC, the present design consists of 50 independent TBA units. Each RK-TBA unit is about $300 \mathrm{~m}$ long, and has 150 extraction cavities ( See Figure 1). To replenish the $360 \mathrm{MW}$ generated from the extraction cavity every $2 \mathrm{~m}$, the $10 \mathrm{MeV}$ drive beam with average current of $600 \mathrm{~A}$ is reaccelerated at $300 \mathrm{kV} / \mathrm{m}$. The design current could be raised slightly to compensate for rf losses in the induction cells (e.g. $625 \mathrm{~A}$ for $4 \%$ loss). The main body of the TBA consists of identical 2-m modules each of which has six $100 \mathrm{kV}$ induction cells and one extraction cavity. Both the drive beam current and the 


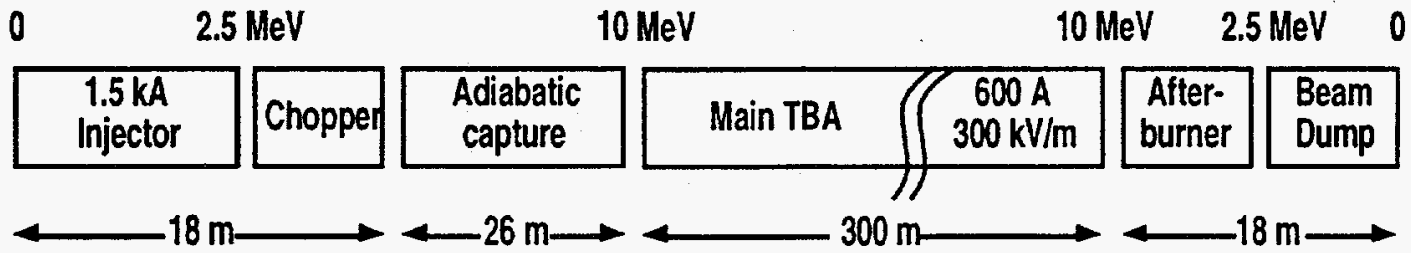

FIGURE 1. Schematic of one RK-TBA unit.

reacceleration voltage have a rise time of $125 \mathrm{~ns}$ and a $200 \mathrm{~ns}$ flat-top, with a falltime that is comparable to the rise time.

The front end of each TBA unit consists of an injector in which $1.5 \mathrm{kA}$ of electrons is generated and accelerated to $2.5 \mathrm{MeV}$. A $5.7 \mathrm{GHz}$ choppertron is placed at this point to generate a bunched beam at $11.4 \mathrm{GHz}$. Chopping reduces the DC current from 1.5 kA to $600 \mathrm{~A}$. This bunched beam at $2.5 \mathrm{MeV}$ is then accelerated to $10 \mathrm{MeV}$ in an 'adiabatic capture section'. Bunching cavities in this section further reduce the length of the bunches, and prepare the beam with the right phase space for injection into the main TBA. At the end of the TBA, there is an 'afterburner' section, in which rf extraction continues, but reacceleration is absent. The afterburner allows us to further extract if power out of the bunched beam, and thus enhances the overall efficiency of the TBA. At the end of the afterburner, the spent beam (at 2 to $3 \mathrm{MeV}$ ) is collected at a beam dump. In the present design, the adiabatic capture section is $25 \mathrm{~m}$ long, while the main TBA has 138 extraction cavities over $276 \mathrm{~m}$. The afterburner consists of 12 rf cavities over $12 \mathrm{~m}$, making a total of 150 extraction cavities.

The overall length of the TBA unit is determined by a balance of two opposing considerations. The longer the TBA unit, the higher the overall efficiency, as the overhead losses from the front and the back ends become a smaller fraction of the total power. On the other hand, the control of beam instabilities and beam degradation effects become increasingly more difficult as the overall length is increased. The longitudinal and transverse beam dynamics simulations to date indicate that the bunched beam can be maintained stably over the $300 \mathrm{~m}$ long TBA unit.

The efficiency for conversion of power in the drive beam to $\mathrm{rf}$ power is easily evaluated for the proposed RK-TBA structure. Allowing for some rf loss in the induction cavities, the overall efficiency is estimated to be $90 \%$. The $10 \%$ loss is shared among the beam loss on the chopper (3.7\%), beam dump (2.8\%) and if into induction cavities (3.6\%). The efficiency for wall plug to drive beam is $40 \%$ (10). Hence the total efficiency from wall plug to rf is $36 \%$.

The rf power requirement of $360 \mathrm{MW}$ per 2 meters determines the product of the accelerating gradient and beam current in the drive beam. Our particular choice of $300 \mathrm{kV} / \mathrm{m}$ and $600 \mathrm{~A}$ is again based on a balance of two considerations. With higher current and lower gradient, the volume of magnetic material required is reduced and the efficiency is increased. However, beam transport becomes more difficult with increasing current.

The linear rise in the drive beam current assures that the extracted $\mathrm{rf}$ field has the right waveform for beam loading in the HGS, while the linear rise in the voltage of the reacceleration cells assure that the entire drive beam stays at 10 $\mathrm{MeV}$ from head-to-tail. This is of course important for both beam transport as well as for rf phase stability. Hence, the rising portion of the current and voltage generate "useful" rf power. But the fall portion is wasted. The corresponding loss 


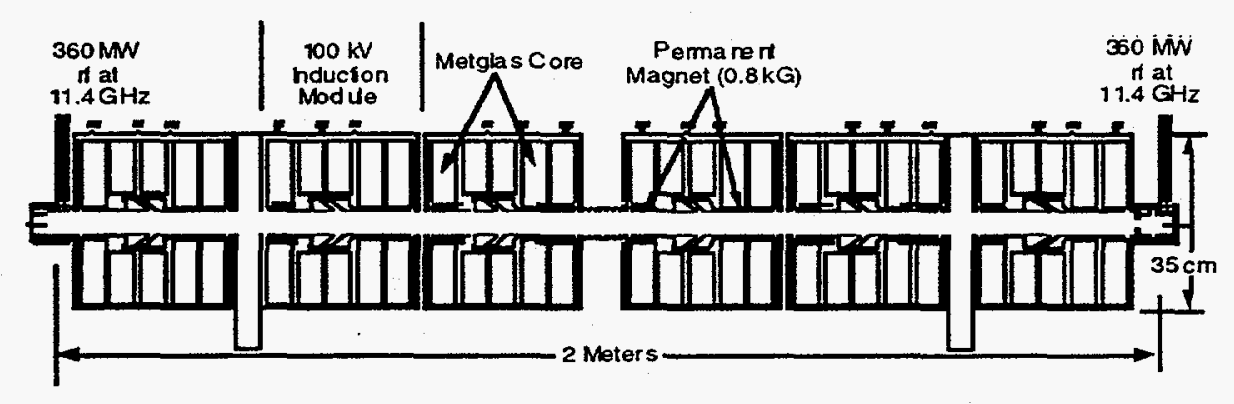

FIGURE 2. Schematic of a 2-m module in the main TBA.

in efficiency due to finite fall time is included in our consideration of the efficiency of the pulse power system (10).

\subsection{Main TBA}

The main TBA consists of identical 2-m modules (Figure 2). The key elements of the module are the permanent quadrupole magnets for focusing of the drive beam, the induction cells for reacceleration, and one rf extraction cavity. Design considerations for each of these major components will be discussed. The key issues in the design are of course related to the physics of if extraction, reacceleration, and transport. However, much attention was paid to make the module compact and efficient. The resulting unit has a diameter of $35 \mathrm{~cm}$. In comparison to other existing induction accelerators, the present design is quite small.

\subsubsection{Pipe Size}

In the design of induction accelerators, the size of the beam tube is usually determined by BBU considerations, since the transverse impedance from the acceleration gap is inversely proportional to the square of the pipe radius. In our TBA design, the low frequency $B B U$ arising from the induction gaps is ameliorated by the low beam current of $600 \mathrm{~A}$, and the Landau damping associated with the large energy spread inherent in the rf buckets. Hence, with proper attention to the gap geometry, we are able to design a narrow tube with a radius of $2.5 \mathrm{~cm}$, and predict acceptable low frequency BBU growth (10).

\subsubsection{Permanent Magnet Focusing}

The small pipe radius allows us to take full advantage of permanent magnets, which have significant cost advantages when the focusing systems are small. Another major reason for the use of permanent magnets is the associated efficiency, as they eliminate the need for power supplies.

Several basic considerations govern the design of the FODO lattice. The foremost requirement is associated with a "trick" concocted to minimize the high frequency $\mathrm{BBU}$ growth arising from the $\mathrm{HEM}_{11}$ mode in the if extraction cavities. 
To suppress the instability growth, we demand that the betatron period be equal to the distance between adjacent extraction cavities, i.e. 2 meters. In addition, the phase advance per lattice period must be less than 90 degrees to ensure beam stability. Finally, the focusing strength must be sufficient to keep the beam envelope small to stay within the beam tube.

The zeroth order design equation is given by the thin lens approximation

$$
\sigma_{0}=\frac{\eta \mathrm{BL}^{2}}{\mathrm{R}[\mathrm{B} \rho]}
$$

where $\sigma_{0}$ is the phase advance per lattice period, $B$ the quadrupole lield strength at pole tip with radial position $R$. $L$ is the half lattice period, and $\eta$ the occupancy factor for the quadupole. The rigidity of the electron beam is given by

$$
[\mathrm{Bp}]=\frac{\beta \gamma \mathrm{mc}}{\mathrm{e}}
$$

where $\beta \gamma m c$ is the momentum of the relativistic beam. $\left[\mathrm{B}_{\rho}\right]$ has the value of $0.035 \mathrm{~T}-\mathrm{m}$ for $10 \mathrm{MeV}$ electrons.

A first order correction for deviations from the thin lens approximation is obtained by replacing the phase advance and occupancy factor with scaled variables given by (11)

$$
\hat{\sigma}_{0}=\sqrt{2\left(1-\cos \sigma_{0}\right)}
$$

and

$$
\eta=\eta \sqrt{2\left(1-\frac{2 \eta}{3}\right)}
$$

In our design, we use $\sigma_{0}$ of 60 degrees. There are 12 half-lattice-periods in 2 meters. The physical occupancy factor is 0.48 . The modified thin lens formula then gives a B-field at pole tip of 800 Gauss. This estimate is within a few $\%$ of the actual $B$ field required to give a $2-m$ betatron period, when we include realistic spatial profile of the magnetic field, as well as the energy variations of the drive beam as it is accelerated over the 2 -m module.

A basic requirement of the transport channel is that the focusing strength must be sufficiently strong to keep the beam from hitting the beam tube. The beam envelope in the focusing channel can be estimated from the equilibrium formula

$$
\frac{\varepsilon^{2}}{a^{2}}=\sigma_{0}^{2}\left(\frac{a}{L}\right)^{2}
$$

where $\varepsilon=\varepsilon_{\mathrm{n}} / \beta \gamma$ and $a$ are respectively the unnormalized edge emittance and the edge radius of the beam envelope. With a normalized edge emittance of $600 \mathrm{~mm}$ $-\mathrm{mr}$, (which is achievable with a good gun design), we obtain an edge radius of about $2 \mathrm{~mm}$ for the beam. 


\subsubsection{Steering and Focusing Corrections}

Steering and focusing corrections are achieved in the present design by means of low-field ( 10 Gauss) DC coils located in the region immediately outside of the permanent magnets, at radial positions of about $4 \mathrm{~cm}$ from axis. These correction coils can be manufactured inexpensively on printed circuit boards.

Since the beam apertures are small, particularly around the extraction cavities, beam centroid displacements must be kept to a minimum. Quad misalignments, when combined with energy variations from head to tail, can lead to beam offsets that change over the length of the pulse, i.e. the "corkscrew" phenomenon. Our strategy for minimizing beam displacements and associated corkscrew is to impose misalignment tolerances that are not excessively tight, design for energy flatness of $<1 \%$, and to correct alignment errors with closely spaced dipole steerers. Detailed steering algorithms have not been worked out yet, but for the purpose of costing, we provide 3 sets of steering coils and two beam position monitors per betatron period.

A key ingredient for the suppression of high frequency BBU is that the betatron period be equal to the distance between adjacent cavities. To ensure that this requirement is obeyed, a feedback system with 2 correction quadrupoles per betatron period is incorporated into the design. A possible monitor for the betatron motion is to use the rf output from the extraction cavities at the dipole frequency. Whether the signal sensitivity is adequate for this purpose needs to be further explored.

\subsubsection{Induction Cores}

The design of induction cells for reacceleration is based on the relation

$$
V \cdot(\Delta t)=A \cdot(\Delta B)
$$

where the core size (with magnetic flux over area $A$ ) is determined by the required voltage $V$ and pulse length $\Delta t$. The allowed flux swing $\Delta B$ is different for different magnetic material. Our present design uses Metglas ${ }^{\mathrm{TM}}$ with $\Delta B$ of 1.3T. Flux swings for Metglas ${ }^{\mathrm{TM}}$ are several times larger than, for example, ferrites, which are commonly used in short pulse induction machines such as ATA and ETA. The cores are correspondingly more compact. Furthermore, Metglas ${ }^{\mathrm{TM}}$ is quite inexpensive, particularly when bought in large quantities.

To determine the transverse dimensions of the core, (with outer radius $R_{\theta}$, and inner radius $R_{i}$ ), we recast Eq. (6) in terms of the accelerating gradient $G$, peak quadrupole field $B$, and core occupancy factor $\eta_{c}$ (fraction of the axial length occupied by induction cores) as follows:

$$
R_{0}-R_{i}=G \Delta t /\left(B \eta_{c}[P F]\right)
$$

where $[\mathrm{PF}]$ is the packing factor, or the fraction of the total core that is occupied by magnetic material, and has a value of 0.65 to 0.75 for typical Metglas ${ }^{\mathrm{TM}}$ cores. Since the accelerating gradient and pulse length are fixed by overall system 
requirements, our design philosophy is to maximize axial core occupancy. The present design has $\eta_{c}$ of 0.75 and a core thickness $R_{o}-R_{i}$ of about $10 \mathrm{~cm}$.

Compact cores reduce cost as well as energy loss. Empirical measurements of core losses (10) can be parametrized to give the following phenomenological formula for the core current (in Amps):

$$
I_{C}=437\left(R_{0}+R_{i}\right) \cdot(\Delta B / \Delta t)^{0.67}
$$

where the radial dimensions are in meters, $\Delta B$ in tesla, and $\Delta t$ in microseconds. The fraction of energy lost in the core $\left(I_{c} / I_{\text {total }}\right)$ is proportional to the size of the core. Our design parameters give a core current of about $400 \mathrm{~A}$. With a $600 \mathrm{~A}$ beam, the core efficiency is $60 \%$.

The magnetic material in a $2 \mathrm{~m}$ module is packaged into 6 independent $100 \mathrm{kV}$ induction cells. Each cell in turn consists of five $20 \mathrm{kV}$ cores. Packaging into small induction cores provides a natural match to the low-voltage pulse power system.

\subsubsection{Pulse Power}

The desired voltage pulse from each core has a $125 \mathrm{~ns}$ linear rise, followed by a $200 \mathrm{~ns}$ flattop at $20 \mathrm{kV}$, followed by a fast fall. This voltage waveform is generated with pulse-forming-networks (PFN's) with tapered impedance to match the induction core. Power input to the PFN's consists of a DC power supply and a Command Resonant Charging unit. The entire pulse power train is at low voltage, and no step-up transformers are needed. Hence, high efficiency and low cost is possible.

\subsubsection{Acceleration Gap}

Each $100 \mathrm{kV}$ induction cell has an induction gap, which is one of the more critical components of the design. The ongoing design efforts (10) evolve around the following key issues:

1. Reduction of high voltage breakdown risks -- To have an induction gap that is safe from breakdown, we need to have sufficiently low gradient across the insulator $(<30 \mathrm{kV} / \mathrm{cm}$ ), sufficiently low fields on the metal surfaces ( < $100 \mathrm{kV} / \mathrm{cm}$ ), proper shielding of the insulators from secondary electrons and $\mathrm{X}$ rays generated by the beam, and proper design of the triple-point (the interface between insulator, metal, and vacuum).

2. Suppression of low frequency BBU -- Dipole modes associated with the cavity (at a few $\mathrm{GHz}$ ) can lead to severe beam-break-up problems if not carefully damped. The required transverse impedance was achieved with heavy de-Qing by placing microwave absorbing material at critical locations around the cavity.

3. Reduction of transverse and longitudinal impedances at high frequencies -- While the high frequency $\mathrm{BBU}\left(\mathrm{HEM}_{11}\right.$ mode at $\left.\sim 14 \mathrm{GHz}\right)$ is generated primarily in the rf extraction cavities, and the major BBU suppression activities center around them, one must be careful to ensure that the transverse impedance contribution from the induction cavities are indeed negligible. In the present 
designs, the transverse impedance around $14 \mathrm{GHz}$ can be made to be lower than half an ohm, and its effect on the high frequency BBU is insignificant. The longitudinal impedance at $11.424 \mathrm{GHz}$ must be low to minimize the microwave power loss. Our design goal is to maintain the induction gap loss to less than $4 \%$, which requires that the longitudinal impedance be less than 4 ohms. Present designs are approaching the required impedance.

\subsubsection{RF Extraction cavities}

Design of the rf extraction is a major ongoing activity in theory and simulations. Present designs evolve around traveling-wave-structures with 3 cells of $8-\mathrm{mm}$ inner radius. The rf output is extracted through 2 separate ports in the 3rd cell, with $180 \mathrm{MW}$ each transported through separate waveguides, and fed directly into the two input couplers of the high gradient structure. Detail design of the cavities center around five key issues:

1. Required output power -- The extraction cavity must have the right effective impedance of about $540 \mathrm{ohms}$ in order to extract $360 \mathrm{MW}$ from a highly bunched beam (70 degrees microbunch length) whose first harmonic current at $11.424 \mathrm{GHz}$ is about $1.15 \mathrm{kA}$.

2. Minimal breakdown risks -- Our present designs use traveling-wavestructures with 3 cells in order to reduce the surface fields. Surface field gradients of less than $70 \mathrm{MV} / \mathrm{m}$ appear possible.

3. Inductive detuning -- In addition to generating the right amounts of power, the rf cavities provide continuous longitudinal bunching for the drive beam. This is accomplished by inductively detuned traveling-wave-structures.

4. Dipole de-Qing -- The rf cavities must have low transverse impedances for the suppression of BBU. Fortunately, there is a natural de-Qing mechanism, as the required extraction cavities have inner radii of around $8 \mathrm{~mm}$, and the dipole modes in the cavity couple to the $\mathrm{TE}_{11}$ mode in the pipe. Simulations to-date suggest that the resulting impedances are sufficiently low for BBU suppression provided that the betatron-node scheme is used in conjunction.

5. Transverse focusing -- rf cavities have transverse fields associated with the fundamental mode. They can degrade the beam envelope if they are sufficiently strong and/or not properly corrected. Evaluation of these transverse forces from theory and simulations is ongoing.

\subsection{Front End and Back End}

Each main TBA unit has a front end that consists of a 'pre-chopper', a chopper, and an 'adiabatic capture' section. At the end of each TBA unit, there is an 'afterburner', followed by a final beam-dump. These five elements will be briefly described.

\subsubsection{Pre-chopper}

The 'pre-chopper' is basically a short induction accelerator to generate an unbunched 300 -ns electron beam at $2.5 \mathrm{MeV}$. This section may consist of an 
injector (at 1 to $1.5 \mathrm{MeV}$ ), followed by a short induction accelerator section that takes the beam to $2.5 \mathrm{MeV}$. The required current from the injector is 1 to $1.5 \mathrm{kA}$, depending on the efficiency of the chopper, which is required to produce at its exit a bunched beam with an averaged current of 600 Amps.

The current waveform required consists of a linear rise over $100 \mathrm{~ns}$, followed by $200 \mathrm{~ns}$ of flat-top. The beam energy should be constant over the entire $300 \mathrm{~ns}$, including the front portion with the rising current. To generate the linearly rising current, the voltage pulse in the $1 \mathrm{MeV}$ injector will also, by the Child-Langmuir Law, have a (nonlinear) $100 \mathrm{~ns}$ rise time. The pulse-forming-network in the subsequent short accelerator section must be arranged to produce a higher voltage at the beam front, thereby compensating for the low energy at injector exit.

Since the electron beam will be transported over $300 \mathrm{~m}$ of narrow pipes, caution must be taken to minimize head-to-tail energy variations, as well as transverse beam offsets and beam temperature. The injector design should produce a bright beam with a normalized edge emittance of $600 \mathrm{~mm}-\mathrm{mr}$ or less, and transverse displacement of 200 microns or less.

\subsubsection{Chopper}

The purpose of the chopper is to generate cleanly separated microbunches at 11.4 GHz. The basic scheme follows the design of Haimson Associates which has been deployed at LLNL. A subharmonic dipole deflecting cavity at $5.7 \mathrm{GHz}$ causes the electron beam to oscillate about a limiting aperture, leading to 'chopped' bunches at twice the oscillating frequency. A similar device can serve as the TBA front end chopper, except that much more effective heat dissipation is required for the $120 \mathrm{~Hz}$ operation of the NLC upgrade.

Since a substantial amount of energy is lost on the chopper, there is incentive to make it efficient. One idea is to precede the subharmonic deflecting cavity with conventional bunching cavities at $11.4 \mathrm{GHz}$. The role of the chopper then is primarily to 'clean up' the particles with the wrong phase. This requires straightforward phasing of the $5.7 \mathrm{GHz}$ input power to the deflecting cavity relative to the incoming pre-bunched beam.

\subsection{3 'Adiabatic Capture'}

The exiting beam from the chopper has microbunch lengths equivalent to $180^{\circ}$ or greater in longitudinal phase space. In the 'adiabatic capture' region, the microbunch lengths are further reduced to $70^{\circ}$ which is the needed bunch length for long-distance propagation in the main TBA. This is accomplished by a number of idler cavities that are more inductive than the rf extraction cavities in the main TBA.

In addition to microbunch sharpening, this section also serves to provide the energy transition from $2.5 \mathrm{MeV}$ to $10 \mathrm{MeV}$. The induction core structures are very similar to the main TBA. However, the voltage waveform should be flat over the $300 \mathrm{~ns}$ of beam-on time, so that the head-to-tail energy flatness required for phase stability could be maintained at $10 \mathrm{MeV}$.

The quadrupole magnets are weaker at the lower energies, and continues to increase with increasing energy, so that the betatron wavelength is kept fixed at 2 
m. Structurally, the 'adiabatic capture' section looks very similar to the main TBA, except that no power is extracted from the idler rf cavities.

\subsection{4 'Afterburner'}

At the end of the TBA is an 'afterburner' section, the primary purpose of which is to increase overall system efficiency by extracting more power out of the nicely bunched beam at the end of the main TBA. This section has a number of rf extraction cavities, permanent magnets for focusing, but no reacceleration cells. The spacings of the rf cavities are changed as the average energy of the beam continues to decrease. The impedance of the cavities are also modified to compensate for changes in the rf bucket. The present design has 12 cavities in the 'afterburner' section, each generating $360 \mathrm{MW}$, and together covering a total of 12 meters.

\subsubsection{Beam-dump}

As the spent electron beam reaches the final beam-dump, its average energy is less than $3 \mathrm{MeV}$. The design of this component is straightforward.

\section{REFERENCES}

1. Sessler, A. M., "The free-electron laser as a power source for a high gradient accelerating structure," Workshop on Laser Acceleration of Particles, NY, AIP Conference Proceedings 91, 1982, pp. 154.

2. Orzechowski, T. J., et al, Phys. Rev. Lett. 57, 2172 (1986).

3. Allen, M. A., et al, Phys. Rev. Lett. 63, 2472 (1989).

4. Westenskow, G. A. and Houck, T. L., "Results of the Reacceleration Experiment: Experimental Study of the Relativistic Klystron Two-Beam Accelerator Concept," presented at the 10th International Conference on High Power Particles Beams, San Diego, CA, June 20-24, 1994, to be published in the Proceedings.

5. Sessler, A. M. and Yu, S. S., "Relativistic Klystron Two-Beam Accelerator," Phys. Rev. Lett., 58, No. 23, 2439-2442 (1987).

6. Clark, J., et. al., "Design and Initial Operation of the ETA-II Induction Accelerator," in Proceedings of the 14 International LINAC Conf., 1988, pp. 19-23.

7. Kapetanakos, C. A. and Sprangle, P., "Ultra-high-current electron induction accelerators," Physics Today 38, 58 (1985).

8. Hogan, W. J., Bangerter, R. and Kulcinski, G. L., "Energy from Inertial Fusion", Physic. Today 42, 9 (1992), pp. 42-50.

9. Yu, S., et al., "Relativistic Klystron Two-Beam Accelerator As A Power Source For A 1 TeV Next Linear Collider - A Systems Study," presented at 17th Int'l LINAC Conf., Japan, August 22-26, 1994, to be published in the Proceedings.

10. Yu, S., Deadrick, F., Goffeney, N., Henestroza, E., Houck, T., Li, H., Peters, C., Reginato, L., Sessler, A., Vanecek, D. and Westenskow, G., "Relativistic Klystron Two-Beam Accelerator As A Power Source For A 1 TeV Next Linear Collider - Design Report," preliminary draft, LBL, Berkeley, CA, September, 1994.

11. Barnard, J., J., et al., "Study of Recirculating Induction Accelerators as Drivers for Heavy Ion Fusion," UCRL-LR-108095, September, 1991. 